\title{
Using clinical practice guidelines in our own backyard: treating our patients with bladder cancer
}

\author{
Peter Black, MD, FRCSC
}

See related article on page 263.

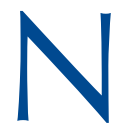
eoadjuvant chemotherapy followed by radical cystectomy has now been established as a standard treatment for invasive bladder cancer since at least 2005, yet it continues to be underutilized. This series from Alberta demonstrates that delivery of chemotherapy has improved from very poor to poor with the introduction of provincial Clinical Practice Guidelines (CPG). ${ }^{1}$ While this improvement must be considered a success, the overriding question remains: why can we not do better? Why were only $18.7 \%$ of patients offered neoadjuvant chemotherapy?

Urologists control the fate of most patients with bladder cancer, and failure of referral to a medical oncologist is the largest obstacle to neoadjuvant chemotherapy. The reasons are presumably manifold, including the belief that the modest efficacy of neoadjuvant chemotherapy does not warrant referral. ${ }^{2}$ A 5\% survival advantage may be celebrated for other cancers, but as urologists we seem hesitant to embrace this for our bladder cancer patients. Some urologists cite the perceived risk that a patient could progress while on chemotherapy if he is among the approximately $40 \%$ of patients who do not respond. Is there also the concern that surgery might be more difficult after chemotherapy?

Do medical oncologists contribute to the low rate of delivery of chemotherapy? Treatment toxicity is more tolerable with the use of gemcitabine and cisplatin instead of methotrexate, vinblastine, doxorubicin and cisplatin (MVAC), but these patients are often older and have multiple medical comorbidities, so that the enthusiasm for administering any chemotherapy is often low. A common trap that we need to avoid is the suggestion that risk-adapted adjuvant chemotherapy may be as effective as neoadjuvant chemotherapy, only to have postoperative therapy withheld due to a lack of evidence to support its use. The goal is not necessarily to treat $100 \%$ of patients with neoadjuvant chemotherapy. Medically high-risk patients should probably proceed with the single most effective therapy (surgery), since they may not be fit for dual therapy. Similarly, if a patient cannot receive cisplatin, surgery should not be delayed by inferior chemotherapy.

One avenue to improve patient care is to enhance the adoption of established guidelines. Do urologists take the time to review the guidelines, and, if so, are they going to be familiar enough with the details if they only see a handful of patients with muscle invasive bladder cancer per year? Treatment of patients at high-volume centres may facilitate closer adherence to established guidelines, although many of the patients in the Alberta study appear to have been treated at such a centre. In a similar manner, multidisciplinary bladder cancer clinics are a promising new approach to improving the quality of care. Some of these questions will be addressed by the Bladder Cancer Quality Care Initiative, a multicentre cooperative effort in the United States and Canada that stems from the Bladder Cancer Advocacy Network "Bladder Cancer Think Tank." Participating centres have agreed to refer all patients with muscle invasive, resectable bladder cancer for preoperative consultation regarding chemotherapy. Reasons for nonreferral and for not proceeding to chemotherapy after referral will be documented prospectively. We should be better able to address the obstacles to optimal treatment after this study.

We do a similarly poor job observing our own guidelines in most aspects of bladder cancer. Single-dose intavesical chemotherapy after transurethral resection of bladder tumour and maintenance bacillus Calmette-Guerin therapy are 2 established standards that we often fail to meet. Re-resection of high-risk non-muscle invasive bladder tumours and extended pelvic lymph node dissections at the time of radical cystectomy are equally important surgical principles that are often ignored. There is much room for improvement.

Assistant Professor, Department of Urologic Sciences, UBC, Vancouver, BC

Competing interests: None declared.

This paper has been peer-reviewed.

\section{References}

1. Miles BJW, Fairey AS, Eliasziw M, et al. Referral and treatment rates of neoadjuvant chemotherapy in muscle-invasive bladder cancer before and after publication of a clinical practice guideline. Can Urol Assoc J 2010;4:263-7.

2. Chung D, Hersey K, Fleshner N. Differences between urologists in United States and Canada in approach to bladder cancer. Urology 2005;65:919-25.

Correspondence: Dr. Peter Black, Department of Urologic Sciences, Faculty of Medicine, UBC, Gordon \& Leslie Diamond Health Care Centre Level 6, 2775 Laurel St., Vancouver, BC V5Z 1M9; fax: 604-875-4637; peter.black@ubc.ca 